\title{
118.
}

\section{ON THE HARMONIC RELATION OF TWO LINES OR TWO POINTS.}

[From the Philosophical Magazine, vol. vi. (1853), pp. 105-107.]

THE "harmonic relation of a point and line with respect to a triangle" is well known and understood ${ }^{1}$; but the analogous relation between two lines with respect to a quadrilateral, or between two points with respect to a quadrangle, is not, I think, sufficiently singled out from the mass of geometrical theorems so as to be recognized when implicitly occurring in the course of an investigation. The relation in question, or some particular case of it, is of frequent occurrence in the Traité des Propriétés Projectives, [Paris, 1822], and is, in fact, there substantially demonstrated (see No. 163); and an explicit statement of the theorem is given by M. Steiner, Lehrsätze 24 and 25 , Crelle, t. XIII. [1835] p. 212 (a demonstration is given, t. XIx. [1839] p. 227). The theorem containing the relation in question may be thus stated.

THEOREM of the harmonic relation of two lines with respect to a quadrilateral. "If on each of the three diagonals of a quadrilateral there be taken two points harmonically related with respect to the angles upon this diagonal, then if three of the points lie in a line, the other three points will also lie in a line,"-the two lines are said to be harmonically related with respect to the quadrilateral.

It may be as well to exhibit this relation in a somewhat different form. The three diagonals of the quadrilateral form a triangle, the sides of which contain the six angles of the quadrilateral; and considering three only of these six angles (one angle on each side), these three angles are points which either lie in a line, or else

1 The relation to which I refer is contained in the theorem, "If on each side of a triangle there be taken two points harmonically related with respect to the angles on this side, then if three of these points lie in a line, the lines joining the other three points with the opposite angles of the triangle meet in a point,"-the line and point are said to be harmonically related with respect to the triangle. 
are such that the lines joining them with the opposite angles of the triangle meet in a point. Each of these points is, with respect to the involution formed by the two angles of the triangle, and the two points harmonically related thereto, a double point; and we have thus the following theorem of the harmonic relation of two lines to a triangle and line, or else to a triangle and point.

Theorem. "If on the sides of a triangle there be taken three points, which either lie in a line, or else are such that the lines joining them with the opposite angles of a triangle meet in a point; and if on each side of the triangle there be taken two points, forming with the two angles on the same side an involution having the first-mentioned point on the same side for a double point; then if three of the six points lie in a line, the other three of the six points will also lie in a line,"-the two lines are said to be harmonically related to the triangle and line, or (as the case may be) to the triangle and point.

The theorems with respect to the harmonic relation of two points are of course the reciprocals of those with respect to the harmonic relation of two lines, and do not need to be separately stated.

The preceding theorems are useful in (among other geometrical investigations) the porism of the in-and-circumscribed polygon.

2 Stone Buildings, July 9, 1853.

C. II. 